\title{
Structural and functional characterization of autophosphorylation in bacterial Histidine Kinases
}

Laura Miguel-Romero ${ }^{\mathrm{a}, 1}$, Cristina Mideros-Mora ${ }^{\mathrm{a}, \mathrm{b}, 1}$, Alberto Marina $^{\mathrm{a}, \mathrm{c}, 2}$ and Patricia Casino ${ }^{\mathrm{d}, \mathrm{e}, 2}$

anstituto de Biomedicina de Valencia, Consejo Superior de Investigaciones Científicas (IBV-CSIC). Jaume Roig 11, 46010-Valencia, Spain

bUniversidad Tecnológica Equinoccial, Facultad de Ciencias de la Salud Eugenio Espejo, Quito, Ecuador

${ }^{\mathrm{C}} \mathrm{CIBER}$ de enfermedades raras (CIBERER-ISCIII), Spain

${ }^{d}$ Departament de Bioquímica i Biología molecular, Universitat de València. Dr Moliner 50, 46100 Burjassot, Spain.

eEstructura de Recerca Interdisciplinar en Biotecnologia i Biomedicina (ERI BIOTECMED), Universitat de València. Dr Moliner 50, 46100 Burjassot, Spain.

${ }^{1}$ Both authors have contributed equally

${ }^{2}$ Corresponding Authors:

Alberto Marina

Instituto de Biomedicina de Valencia (CSIC). Jaume Roig 11, Valencia 46010, Spain

E-mail: amarina@ibv.csic.es

Tel: +34963391754;Fax: +34963690800

Patricia Casino

Universitat de València-ERI Biotecmed

E-mail: patricia.casino@uv.es

Tel: +34-963543020; Fax: +34-96 3544635

Running Head: Histidine kinase autophosphorylation 


\section{Abstract}

Autophosphorylation of histidine kinases $(\mathrm{HK})$ is the first step for signal transduction in bacterial two-component signalling systems. As HKs dimerize, the His residue is phosphorylated in cis or trans depending on whether the ATP molecule used in the reaction is bound to the same or the neighbouring subunit, respectively. The cis or trans autophosphorylation results from an alternative directionality in the connection between helices $\alpha 1$ and $\alpha 2$ in the HK DHp domain, in such a way that $\alpha 2$ could be oriented almost $90^{\circ}$ counter-clockwise or clockwise with respect to $\alpha 1$. Sequence and length variability of this connection appears to lie behind the different directionality and is implicated in partner recognition with the response regulator $(R R)$, highlighting its importance in signal transduction. Despite this mechanistic difference, $\mathrm{HK}$ autophosphorylation appears to be universal, involving conserved residues neighbouring the phosphoacceptor His residue. Herein, we describe a simple protocol to determine both autophosphorylation directionality of $\mathrm{HKs}$ and the roles of the catalytic residues in these protein kinases.

Keywords: Histidine kinases, two-component systems, cis- transautophosphorylation, signal transduction, heterodimer production and purification, X-ray crystallography. 


\section{Introduction}

Bacterial histidine kinases (HKs) are signal transduction enzymes involved in the sensing of different stimuli and transmitting changes in those stimuli to the inner cell [1]. Sensing and transmitting signals by HKs imply ATP uptake, autophosphorylation and phosphoryl transfer. In recent years, autophosphorylation of HKs has drawn attention as the structural and functional features governing this enzymatic activity have been better characterised. HKs are dimeric proteins and comprise a catalytic core formed by two domains joined by a flexible linker. One domain, called DHp (Dimerization and $\underline{H}$ istidine phosphotransfer), is formed by two antiparallel $\alpha$-helices ( $\alpha 1$ and $\alpha 2$ ) connected by a loop, while the other domain, CA ( $\underline{C}$ atalytic and $\underline{A}$ TP-binding), is globular with an $\alpha / \beta$ sandwich fold that binds ATP [2, 3] (Figure 1). The DHp domain holds the phosphoacceptor His at helix $\alpha 1$ and mediates dimerization into a four helix bundle merging two subunits into a dimer (Figure 1). In that way, the CA domain that binds ATP can approach the phosphoacceptor His in either subunit of the dimer, enabling autophosphorylation [4]. If the CA domain bound to ATP approaches the phosphoacceptor His within the same subunit, the HK adopts the so called "cis" autophosphorylation conformer, while if it approaches the His of the other subunit the HK adopts the "trans" autophosphorylation conformer [5-7] (Figure 1). Structural and functional evidence suggests that the connection between helices $\alpha 1$ and $\alpha 2$ in the $\mathrm{DHp}$ domain could play a key role regulating the directionality of autophosphorylation $[7,8,9]$. Therefore, it has been hypothesized that the length and residue composition of this link (Figure 2) would select between cis or trans autophosphorylation [10]. Crystal structures of DHp domains from HKs whose autophosphorylation has been confirmed 
biochemically to occur in cis (i.e. HK853 from Thermotoga maritima [7]) or in trans (i.e. EnvZ and CpxA from E. coli $[11,12])$ show that the helix $\alpha 2$ in the DHp domain is connected almost $90^{\circ}$ counter-clockwise or clockwise with respect to $\alpha 1$, respectively (Figure 3). Interestingly, the alternative directionality adopted by HKs is independent of the catalytic mechanism for the autophosphorylation reaction, which has been demonstrated to be conserved [10]. Thus, the change in directionality at the connection between helices $\alpha 1$ and $\alpha 2$ could play a different role in two-component systems (TCS) signalling. Since the DHp $\alpha 1-\alpha 2$ connection is one of the regions recognized by the RR in the interaction with the HK, alternative directionalities could be critical in preserving the exquisite fidelity showed by the HK-RR pairs in TCS, avoiding cross-talk between phosphate donors and acceptors, which could compromise bacterial survival. Here, we will outline methodology that can be used to unveil if an HK works by a cis or trans autophosphorylation reaction, which should be directly related with the directionality of the connection between helices $\alpha 1$ and $\alpha 2$ at the DHp domain. Moreover, we will dissect the catalytic mechanism for the autophosphorylation reaction through the analysis of the most relevant catalytic residues. These methodologies will be broadly applicable for the characterisation of structural and functional features of other HKs that govern their autophosphorylation.

\section{Materials}

\subsection{Cloning}

The proteins used for all the experiments should correspond to the catalytic domains of HKs, comprising only the DHp and CA domains. To locate the catalytic domains of the HK under study, computational tools such as SMART 
[13] and Pfam [14] can be used. For example, the catalytic domain of HK853 of Thermotoga maritima is comprised from residues 232 to 489 , while residues 224450 constitute the HK of EnvZ from E. coli.

1. Chemically synthesized DNA primers to amplify the gene of a HK are prepared at $10 \mu \mathrm{M}$ dissolved in water or in Tris-EDTA buffer (TE buffer: $10 \mathrm{mM}$ Tris-HCl pH 8.0 and $1 \mathrm{mM}$ EDTA) (see Note 1 and 2).

2. Bacterial expression plasmids: $5-50 \mathrm{ng}$ dissolved in water or in Tris-EDTA buffer (see Note 3).

3. Agarose gel solution: $1 \%$ agarose dissolved in $1 \times$ TAE buffer ( $40 \mathrm{mM}$ Tris, $20 \mathrm{mM}$ Acetate and $1 \mathrm{mM}$ EDTA) and heat in the microwave till completely dissolved. For a $100 \mathrm{ml}$ gel add $2 \mu \mathrm{L}$ of GreenSafe Premium (NZYtech, Portugal) before loading solution in casting tray with comb.

4. PCR or gel cleaning kit (eg. NucleoSpin Gel and PCR Clean-up, MachereyNagel)

5. Dpnl restriction enzyme (Clontech).

6. T4-DNA polymerase (Thermo Fisher Scientific) (see Note 4)

7. Competent cells of $E$. coli strain $\mathrm{DH} 5 \alpha$; use $50 \mu \mathrm{L}$ of electro-competent cells prepared in $10 \%$ glycerol (see Note 5 ).

8. Luria-Bertani (LB) agar plates: Weigh $10 \mathrm{~g}$ of tryptone, $5 \mathrm{~g}$ of yeast extract, 10 $\mathrm{g}$ of $\mathrm{NaCl}$ and $15 \mathrm{~g}$ bacteriological agar and make up to $1 \mathrm{~L}$ in water. Autoclave and when the medium is under $50^{\circ} \mathrm{C}$ add the corresponding antibiotic, typically ampicillin at $100 \mu \mathrm{g} / \mathrm{ml}$ or kanamycin at $33 \mu \mathrm{g} / \mathrm{ml}$; mix well and dispense in agar plates.

9. Primers flaking the plasmid-multicloning site.

2.2 Site-directed mutagenesis 
It was performed following the Quickchange method developed by Stratagene (La Jolla, CA).

1. High fidelity DNA polymerase such as KOD Hot Start DNA polymerase (Merck Millipore).

\subsection{Protein expression}

1. For cell growth, prepare Luria-Bertani (LB) broth: weigh $10 \mathrm{~g}$ of tryptone, $5 \mathrm{~g}$ of yeast extract, $10 \mathrm{~g}$ of $\mathrm{NaCl}$ make up to $1 \mathrm{~L}$ in water, autoclave and when the medium is under $50^{\circ} \mathrm{C}$ supplement with corresponding antibiotics (Ampiciline $100 \mu \mathrm{g} \mathrm{ml}^{-1}$, Chloramphenicol $33 \mu \mathrm{g} \mathrm{ml}^{-1}$ and/or Kanamycin $33 \mu \mathrm{g} \mathrm{ml}^{-1}$, depending on the cell strain and the plasmid used in each case).

2. Spectrophotometer to measure $O D$ at $600 \mathrm{~nm}$

3. Isopropil- $\beta$-D-1-tiogalactopiranósido (IPTG) at $1 \mathrm{M}$ stock solution or the corresponding inductor to start the protein expression.

\subsection{Protein purification}

1. Use ultrasonic processor or emulsifier.

2. Lysis buffer (buffer A): $50 \mathrm{mM}$ Tris- $\mathrm{HCl} \mathrm{pH} 8.0$ and $500 \mathrm{mM} \mathrm{NaCl}$. Use a pH according to the protein pl. Add $1 \mathrm{mM}$ of phenylmethylsulfonyl fluoride (PMSF) to inhibit protease activity and $1 \mathrm{mM}$ of $-\beta$-mercaptoethanol to avoid oxidation (see Note 6).

3. Affinity chromatography columns, depending on the tag fused to the protein (e.g. $1 \mathrm{ml}$ or $5 \mathrm{ml}$ HisTrap columns (GE Healthcare) for Histag-proteins).

4. Elution buffer (buffer B): buffer A supplemented with a specific competitor ligand to dissociate the protein bound to the affinity column (e.g. imidazole at 300500 mM for HisTrap columns).

5. Gel filtration buffer: $50 \mathrm{mM}$ Tris- $\mathrm{HCl}, \mathrm{pH} 8.0$ and $150 \mathrm{mM} \mathrm{NaCl}$ 
6. Gel filtration column: HiLoad 16/60 Superdex 200 (Ge Healthcare)

7. Perform SDS-PAGE using $15 \%$ gel with an acrylamide:bisacrylamide ratio (20:1) for a HK with a MW 30KDa.

8. Loading buffer: mix $3,55 \mathrm{ml}$ of deionized water, $1,25 \mathrm{ml}$ of $0,5 \mathrm{M}$ Tris $\mathrm{pH} 6,8$, $2,5 \mathrm{ml}$ of glycerol, $2 \mathrm{ml}$ of $10 \%$ SDS and $0,2 \mathrm{ml}$ of $0,5 \%$ Bromophenol blue; homogenize, take $950 \mu \mathrm{l}$ and add $50 \mu \mathrm{l}$ of $\beta$-mercaptoethanol. The ratio of sample:loading buffer is $2: 1$.

9. Centrifugal filters such as Amicon Ultra (Millipore).

10. ÄKTA Purification System (GE Healthcare).

11. SnakeSkin ${ }^{\mathrm{TM}}$ Dialysis Tubing, 3.5K MWCO (ThermoScientific).

\subsection{HK phosphorylation}

1. Buffer A supplemented with $100 \mathrm{mM} \mathrm{KCl}_{2}$ and $10 \mathrm{mM} \mathrm{MgCl}_{2}$.

2. Mixture of cold (0.1-0.3 mM) ATP and hot $\left(0.1 \mathrm{mCi} \mathrm{m}^{-1}\right)$ ATP- $\left[\gamma^{32}\right]$ ATP $\left(3000 \mu \mathrm{Ci} \mathrm{mmol}{ }^{-1}\right)$ diluted in deionized water. It is recommended to prepare a $10 \mathrm{X}$ cocktail to add same amounts to all samples.

3. Stop loading buffer: SDS-PAGE loading buffer containing 2x SDS detergent and $50 \mathrm{mM}$ de EDTA.

4. Gel blotting and drying: use 3MM blotting paper to transfer the gel followed by incubation into a gel drying system under vacuum (Bio-Rad Model 583 Gel Dryers and Hydrotech pump).

5. Phosphorylated proteins are visualized by autorradioagraphy using the specific equipment, for example Fluoro Image Analyzer FLA-5000 (Fuji) and analysed with the associated software (MultiGauge software, Fuji). 


\subsection{Crystallization and crystal freezing}

1. Commercial crystallization screens (at least three). We recommend start with JBScreen Classic HTS I+II (from Jena Bioscience) and MIDAS (from Molecular Dimensions).

2. MRC 2-well or 3-well crystallization plates (Molecular Dimensions) that allow to try crystallization of the protein alone and in the presence of ligands such as ATP , ADP or the non-hydrolyzable ATP analogs AMPPNP or AMPPCP.

3. Liquid nitrogen is used for crystal freezing.

4. Cryo loops for crystal mounting (MiTeGen or Hampton Research)).

5. X-ray data processing programs (for example, IMosflm from CCP4 [15]).

6. Software suites for automated macromolecular structure determination by Xray crystallography (for example, CCP4 [15] and PHENIX [16] or equivalent).

7. Macromolecular visualization software (Coot [17] and PYMOL [18] or equivalent).

\section{Methods}

\subsection{Determination of the autophosphorylation directionality in HKs}

Due to the dimeric nature of HKs, the autophosphorylation reaction could take place within the same (intra-)subunit or between (inter-)subunits driving a cis or trans phosphorylation in the homodimer, respectively (Figure 1). To find out which directionality is driving a specific $\mathrm{HK}$, it is necessary to discern which subunit is phosphorylated within the homodimer and which is the one that phosphorylates. For this propose, heterodimers containing two subunits with different length, a Short (S) and a Long (L) version of the catalytic domains should be produced. Furthermore, to unambiguously distinguish the phosphorylated subunit in the 
dimer, two additional types of mutant heterodimers are required. One type contains a mutation at the CA domain just in the $L$ subunit, impairing ATP-binding ( $L^{\varnothing ; ~ k i n a s e ~ m u t a n t), ~ a n d ~ p r o d u c i n g ~ t h e ~} L^{\varnothing} S$ heterodimer. The other type contains subunit $L^{\varnothing}$ and a mutation at the phosphoacceptor His in the $S$ subunit $\left(\mathcal{S}^{\varnothing}\right.$; phosphoacceptor mutant), imparing its capacity to be phosphorylated, and producing $\mathrm{L}^{\varnothing} \mathrm{S}^{\varnothing}$ heterodimer (Figure 4A).

\subsubsection{Cloning of HKs constructs to generate $L$ and $S$ subunits.}

To generate subunits $L$ and $S$ with different length, genes for the specific HKs must be cloned into two plasmids, one containing a tag (e.i. His-tag) and the other without tag (or with tag of different size). Plasmids containing tag, or the longest tag will produce a longer HK protein, defined by L, while the plasmids devoid of tag or with the shortest tag will produce shorter protein defined by S.

1. Amplify the genetic region of a specific HK construct by carrying on a Polymerase Chain Reaction (PCR) according to the protocol recommended for the High fidelity DNA polymerase used (e.g. for $25 \mu \mathrm{lmix} 5-50 \mathrm{ng}$ of DNA template with the polymerase Buffer $1 \mathrm{X}$, specific designed primers and dNTPs at $0.2 \mu \mathrm{M}$ final concentration, $2 \mathrm{mM} \mathrm{MgSO}_{4}$ and $1 \mathrm{U}$ of High fidelity DNA polymerase. Then incubate $10 \min 95^{\circ} \mathrm{C}$ for denaturalization followed by 35 cycles of $30 \mathrm{sec}$ at 95 ${ }^{\circ} \mathrm{C}, 30 \mathrm{sec}$ at primer annealing temperature and extension (30 sec/Kb) at $72{ }^{\circ} \mathrm{C}$. A final extension of $5-10$ min at $72^{\circ} \mathrm{C}$ could be added. See Note 1.

2. Plasmid linearization will vary depending on the cloning techniques used (e.g. LIC cloning, In-fusion or enzyme restriction), thus, use the appropriate technique. 3. Load the PCR products and linearized plasmids into a 1-2\% agarose gel to visualize the correct size for the amplified regions and linearized plasmids. Cut 
the corresponding gel bands with a scalpel and purify the DNA with a gel cleaning kit following the manufacture's instructions. Linearized plasmids could also be directly cleaned with a PCR clean-up kit without the electrophoretic step.

4. For LIC cloning, treat with T4 polymerase the amplified genes and linearized plasmid. For a $10 \mu \mathrm{l}$ reaction: $5 \mu \mathrm{L}$ sample, $2.5 \mathrm{mM}$ of corresponding dNTP, 0.1 $\mathrm{mg} / \mathrm{ml} \mathrm{BSA}, 1 \times$ T4 polymerase buffer, 5mM DTT (1,4-Dithiothreitol) and $0.5 \mu \mathrm{L}$ of T4-DNA polymerase. Incubate reaction in PCR machine or thermoblock: 25 min $22^{\circ} \mathrm{C}$ followed by $20 \min 75^{\circ} \mathrm{C}$ for enzyme inactivation and store at $4^{\circ} \mathrm{C}$.

5.Then, mix them in a 2:1 ratio (gene:plasmid), incubate at room temperature (RT) for $5 \mathrm{~min}$ and put them on ice. For In-fusion cloning, mix amplified genes and plasmids in a 2:1 ratio with $5 \mathrm{X}$ In-Fusion HD Enzyme Premix, incubate $50^{\circ} \mathrm{C}$ for 15 min and put on ice.

6. Immediately after step 4 transform $3 \mu$ of cloning mixture by electroporation in $50 \mu \mathrm{l}$ of DH5 $\alpha$ cells. Then, add $1 \mathrm{ml}$ of cold LB to the cells followed by incubation at $37^{\circ} \mathrm{C}$ with shaking during $1 \mathrm{~h}$.

7. Centrifuge the tube at $6000 \times g$ for 2 min, discard the media without touching the cells. Resuspend slowly the cells in the same media; spread all on a plate with the corresponding antibiotics and grow overnight $(\mathrm{o} / \mathrm{n})$.

8. Check if the cloning was successful by picking the colonies grown in the plate and performing a PCR assay (DNA template comes from the colony) using primers flaking the plasmid-multicloning site. Confirm the correct cloning by sequencing PCR products coming for those colonies which show bands of the appropriated molecular size.

9. For further mutagenesis or protein expression analysis, purify plasmid DNA of the positive colonies. 


\subsubsection{Mutant design}

The chosen point mutations are a key factor in this methodology in order to produce subunits that cannot be phosphorylated and subunits that cannot phosphorylate. To obtain a subunit that cannot be phosphorylated, point mutations at the phosphoacceptor His should be introduced in the plasmids devoid of tag or with the shortest tag which can be defined as constructs $S^{\varnothing}$ or named as phosphoacceptor mutant. Meanwhile to produce a construct that cannot phosphorylate, mutations that impair ATP binding at the CA domain (see Note 7) could be introduced in the plasmids containing the tag or the longest tag, which can be defined as constructs $L^{\varnothing}$ or named as kinase mutant (see Note 8). 1. Mutagenesis is performed by the Quickchange method developed by Stratagene (La Jolla, CA). A PCR is carried out with the wild type HKs plasmids and the appropriate primers as described in the section 3.1.1.1 but using longer extension times in order to introduce the mutation while amplifying the whole plasmid.

2. To remove methylated parental plasmid, digest de PCR product with $1 \mu \mathrm{l}$ of Dnpl at $37^{\circ} \mathrm{C}$ for $2 \mathrm{hrs}$ or the time indicated by supplier.

3. Continue with transformation as described in 3.1.1.5.

4. Check if the colonies contain the plasmid with the specific mutation by extracting the DNA and sequencing.

\subsubsection{Homo and heterodimer production}

To produce homodimer and heterodimer species of a HK, similar steps related with expression and purification are followed. However, in the case of heterodimer production other methodologies, besides the co-expression method, 
can be used taking advantage of the spontaneous subunit exchange peculiarity of some HKs (see Note 9) [11].

1. For homodimer production, transform by electroporation $20 \mathrm{ng}$ of the corresponding plasmid in $50 \mu \mathrm{L}$ of expression cells. For heterodimer production, co-transform $20 \mathrm{ng}$ of each plasmid in $50 \mu \mathrm{L}$ of expression cells. To determine the autophosphorylation directionality using heterodimers, three different species of heterodimers LS, $L^{ø} S$ and $L^{ø} S^{\varnothing}$ should be generated. For that purpose, three different co-transformations should be performed for each HK using two different plasmids containing the appropriate constructs; i) (L) and (S) containing wild type constructs, ii) $\left(L^{\varnothing}\right)$ and $(S)$ containing mutation at the long construct, iii) $\left(L^{\varnothing}\right)$ and $\left(S^{\varnothing}\right)$ containing mutations at both long and short constructs (Figure 4A). Notice that plasmids for the $L$ and $S$ versions of the protein must confer resistance to different antibiotics (see Note 10).

2. Add $1 \mathrm{ml}$ of cold $\mathrm{LB}$ to the cells followed by incubation at $37^{\circ} \mathrm{C}$ with shaking during $1 \mathrm{~h}$.

3. Spread 100-200 $\mu \mathrm{l}$ on a plate with the corresponding antibiotics (plasmid and cells) and grow overnight (o/n).

4. To generate a bacterial glycerol stock for future protein expressions, inoculate one colony in $5 \mathrm{ml}$ of LB with the corresponding antibiotics and grow o/n. Next day, mix $800 \mu$ of saturated culture with $400 \mu$ of sterile $50 \%$ glycerol and save it at $-80^{\circ} \mathrm{C}$.

\subsubsection{Protein expression}

1. To recover the bacteria from the glycerol stock, scrape some of the frozen bacteria and streak it into a LB plate with the corresponding antibiotics and incubate at $37^{\circ} \mathrm{C} \mathrm{o} / \mathrm{n}$. 
2. Inoculate one colony in $20 \mathrm{ml}$ LB with appropriate antibiotics and grow o/n in a shaker at $37^{\circ} \mathrm{C}$ to obtain a saturated culture.

3. Inoculate $20 \mathrm{ml}$ of $\mathrm{o} / \mathrm{n}$ saturated culture into $1 \mathrm{~L} \mathrm{LB}$ (for a 1/50 dilution) supplemented with the appropriate antibiotics.

4. When the culture has reached exponential phase $\left(\mathrm{OD}_{600} \sim 0.6\right)$, protein expression is induced by addition of IPTG (1 $\mathrm{ml}$ of $1 \mathrm{M}$ stock solution) or the corresponding inductor.

5. After $3 \mathrm{~h}$ of induction at $37^{\circ} \mathrm{C}$, cells are collected by centrifugation at $5000 \times \mathrm{g}$ for $15 \mathrm{~min}$ in a refrigerated centrifuge. Then, discard the supernatant and flashfreeze the pellets in $\mathrm{LN}_{2}$ and store at $-80^{\circ} \mathrm{C}$.

\subsubsection{Homodimer purification}

Protein purification steps are carried at $4^{\circ} \mathrm{C}$.

1. When ready to proceed with the purification, resuspend the cell pellets in cold lysis buffer (around 40-50 $\mathrm{ml}$ for the pellet coming from a $1 \mathrm{~L}$ culture). Make sure that the suspension is completely homogenized with no clumps (see Note 11).

2. Disrupt the cells by sonication or other ways (French press), centrifuge for 30 min at $16000 \times g$ and collect the supernatant containing the overexpressed proteins.

3. The clarified supernatant is loaded into a $1 \mathrm{ml}$ affinity column (corresponding with the tag fussed to the protein) previously equilibrated with buffer A.

4. In the case of His-tag, the column is washed with $7-8 \%$ of buffer B containing $0.5 \mathrm{M}$ imidazole to remove unspecific binding and proteins are eluted using 40 $70 \%$ of buffer $\mathrm{B}$. If other tag is used, elute the protein following the affinity column recommendations.

5. Additional gel filtration chromatography can be performed as a final step. 


\subsubsection{Heterodimer purification}

To purify the heterodimers species, follow the initial steps described in 3.1.5 for cell disruption. Then, use the clarified supernatant for further affinity chromatography steps keeping in mind the nature of the fusion tags in the heterodimer. As an example, affinity chromatography using a $5 \mathrm{ml}$ HisTrap column was performed to purify a heterodimer containing one His-tag in the $\mathrm{L}$ subunit. A 20 -column volume gradient from 0 to $30 \%$ of buffer B was used in order to separate individual $L$ or $S$ constructs from the heterodimer as can be shown in Figure 4B.

\subsubsection{Autophosphorylation assay}

The visualization of autophosphorylation in a dimeric $\mathrm{HK}$ allows identifying a cis or trans directionality in the reaction.

1. Prepare $10 \mu \mathrm{l}$ of the phosphorylation mixture with $4 \mu \mathrm{M}$ of the homo or heterodimer HK and $1 \mu \mathrm{l}$ of autophosphorylation buffer. Adjust to $9 \mu \mathrm{l}$ with water. 2. The reaction is carried out at room temperature and starts with the addition 1 $\mu$ l of the 10 X ATP cocktail which helps to add same amounts to all samples.

3. After 5-30 min, depending on the autophosphorylation capacity of the analysed HK (e.g. 10 min for HK853 and EnvZ), reactions are stopped by the addition of loading buffer containing 2x SDS in a ratio 2:1 (Sample:loading buffer). Important: boiling the samples could lead to the loss of the phosphorylated radioactive label, therefore, samples should be maintained at room temperature for $15 \mathrm{~min}$ before performing electrophoresis.

4. Then, load $8 \mu$ l of the reaction samples in a $15 \%$ SDS gel and run the gel at 200 volts until the bromophenol blue indicator marker arrive to the bottom of the gel. 
5. Extract the gel and transfer it to a 3MM blotting paper and dry the gel.

6. Visualize the phosphorylated proteins by autorradioagraphy using the available equipment and analyse phosphorylation of the dimers using the appropriate software.

The cis autophosphorylation is confirmed by the lack of radioactive signal in the heterodimer $L^{\varnothing / S^{\varnothing}}$ and phosphorylation signal only in the $S$ subunit of the heterodimer $L \varnothing / S$. On the other hand, the trans autophosphorylation could be determined visualizing the heterodimer $\left(L^{\varnothing / S^{\varnothing}}\right)$ where $L^{\varnothing}$ is the only phosphorylated subunit since the reaction takes place between subunits. A scheme of the heterodimers hypothetical pattern of autophosphorylation and the experimental results obtained with HK853 (cis) and EnvZ (trans) are shown in Figure 4C.

Cis or trans autophosphorylation has been related to the connection between helices $\alpha 1$ and $\alpha 2$ at DHp domain, a connection which is variable in sequence and length as it is observed by the 3D structures of this domain from different HKs (EnvZ, CpxA, VicK and HK853) (Figure 2). Thus, the interchange of this connection with a HK showing a distinct autophosphorylation pattern could be a useful strategy to provide clues on the interaction specificity for response regulator recognition as it has been described by Casino $\mathrm{P}$. et al [10].

\subsection{Visualizing the autophosphorylation reaction}

To visualize the molecular basis of the autophosphorylation reaction, crystallization studies can be performed (Figure 5). The structure of a homodimeric HK performing the autophosphorylation reaction not only would provide clues for the directionality of this reaction but also would provide clues on 
the reaction mechanism that can be further confirmed by a mutagenesis study. For this propose, the structures of different HKs have been solved by X-ray crystallography $[10,12]$.

\subsubsection{HK expression and purification}

1. The HK can be expressed and purified following the general affinity chromatography protocol described in 3.1.4-5 section or alternative protocols.

2. To increase the probability of crystallization, we recommend to remove the tag fused to the HK by proteolytic cleavage adding a specific protease in a protein:protease ratio typically used from $50: 1$ to $20: 1$. For certain proteases, such as Tobacco Etch Virus Protease (TEV) or Human Rhinovirus 3C Protease (3C / PreScission) addition of $0,5 \mathrm{mM}$ EDTA and $5 \mathrm{mM} \beta$-mercatoethanol increases cleavage efficiency. The protein-protease mixture can be incubated at $4^{\circ} \mathrm{C}$ overnight and perform dialysis in buffer $\mathrm{A}$ at the same time to remove the competitor ligand (imidazole, glutathione, maltose...) for a further second affinity chromatography step (Figure 5A).

3. The cleaved protein was purified by a second affinity chromatography where the uncleaved protein is retained. The unretained protein is pooled and concentrate to $<2 \mathrm{~mL}$ using Amicon centrifugal filters. A final gel filtration chromatography purification step over Superdex 200 column equilibrated with gel filtration buffer is recommended to eliminate aggregated forms of the protein.

4. Pool peak fractions and concentrate to $10-20 \mathrm{mg} / \mathrm{mL}$ using Amicon centrifugal filters.

3.2.2 Protein Crystallization, data collection and 3D structure determination.

To identify crystallization conditions vapour diffusion method using sitting drops is the most commonly method used [19]. The use of a liquid handing system (e.g. 
Mosquito by ttplabtech) that allow low volume drops (0.1- $0.2 \mu \mathrm{l})$ is recommended in order to carry out more crystallization assays with the minimal volume of sample.

1. Set up crystallization assays by depositing in 96-weel plates equal amounts (e.g. $0.2 \mu \mathrm{l}$ ) of purified $\mathrm{HK}$ at $10-20 \mathrm{mg} / \mathrm{ml}$ and crystallization screen conditions. Reservoirs are filled with 50-75 $\mu \mathrm{l}$ of the corresponding crystallization condition. Once the 96 screen conditions are set up, the plate is seal with a transparent film and is incubated at an appropriate temperature, usually 21 or $4 \stackrel{\circ}{ } \mathrm{C}$ (Figure $5 \mathrm{~B}$ ).

2. Screenings are monitored daily during the first week to detect crystal growth, then, can be monitored once a week to obtain a hit condition.

3. Optimize the initial crystallization conditions by varying $\mathrm{pH}$, salt and precipitant concentration around the original hit conditions. Another option is the use additive screenings.

4. Once the conditions producing single crystals are found, scale up the volume of the crystallization assay $(0.5-2 \mu \mathrm{l}$ for the drop and $0.5-1 \mathrm{~mL}$ for the reservoir) to produce crystals of higher size.

5. Test cryoprotectant capacity of the crystallization conditions by flash freezing this solution in liquid nitrogen and taking some X-ray diffraction snapshots. The absence of concentric rings characteristics of ice formation in the diffraction image will indicate that the solution is a good cryoprotectant. If ice-rings are observed, include cryoprotectant in the crystallization conditions and soak the crystals in this solution from $10 \mathrm{~s}$ to $2-3 \mathrm{~min}$, and then flash freeze in liquid nitrogen (see Note 12).

6. Collect complete X-ray diffraction data sets and solve the structure by molecular replacement using as template one of the HK structures available in 
the PDBs (Figure 5C). Pipelines for automated structure solution using molecular replacement are available in the CCP4 Suite [20] (such as Balbes, MrBump and MoRDa) or PHENIX Suite [21] as well as in the Auto-Rickshaw web server [22].

\subsubsection{Functional characterization of autophosphorylation reaction}

In the light of the structural data obtained for the autophosphorylation reaction, several potential catalytic residues for this reaction can be identified (Figure 6A). Thus, to assess the effect of these residues in HK autophosphorylation, a mutagenesis study together with in vitro phosphorylation assays can be performed (see Note 13).

The results of these assays can be compared with the mutagenic characterization of other HKs showing cis or trans autophosphorylation (e.g. HK853 showing cis and EnvZ showing trans as models).

1. Identify potential catalytic residues in the active site of the 3D structure by analysing the contacts of the substrate ATP (or a non-hydrolysable analogue) and the phosphoacceptor His, as well ions or water molecules to which they are coordinated. Compare the active center organization with the active center of other HKs previously characterized. It could be interested to introduce mutations at the phosphoacceptor His as well as at the conserved acidic residue after the His, if it is present (Figure 6A). Likewise, introduce a mutation at a conserved polar residue in a4 which stabilizes the interaction between the two previous residues (the phosphoacceptor His and the acidic residue).

1. Site-directed mutagenesis of potential catalytic residues can be generated as described in section 3.1.2.

2. The mutants are expressed and purified as described in section 3.1.4-5. 
3. Carry out autophosphorylation assays as it is described in section 3.1 .7 and quantify the effect of the individual mutations for this activity. Compare the effect of these mutations with similar mutations reported in other HKs showing cis and trans authophosphorylation such as HK853 and EnvZ (Figure 6A). For HK853 and EnvZ, mutations at the conserved acidic residue completely abolished phosphorylation highlighting the relevance of this residue in the autophosphorylation mechanism (Figure 6B). Mutations at the conserved polar residue in $\alpha 4$ had a deleterious impact in the autophosphorylation demonstrating its role stabilizing the interaction between the phosphoacceptor His and the acidic residue (Figure 6B). Additional mutations at other specific residues, such as mutation at the conserved Asn in a5 in charge of chelating the $\mathrm{Mg}^{2+}$ cation, completely abolished phosphorylation due to impair binding of ATP at the active center (Figure 6B). Also, mutations in the conserved basic residues that contact the $\beta$ and $\gamma$ phosphates of ATP showed low phosphorylation levels mainly related with a decreased ATP binding level and/or the stabilization of the negative charge produced in the ADP product (Figure 6B). Similar results obtained for the HK of study would demonstrate that it presents a canonical autophosphorylation mechanism [10]. If differences in the effect of the mutations are observed would suggest a different mechanism of autophosphorylation and a deeper characterization of the $\mathrm{HK}$ would be required in order to identify molecular peculiarities.

\section{Notes}

Note 1. HK DNA can be obtained by PCR using as template the genomic DNA of the corresponding bacteria or from a synthetic gene of the HK. Synthetic genes 
can be designed using codon optimization to enhance protein expression in $E$. coli.

Note 2. Primers should be designed such that they incorporate the overhang necessary to subclone the HK cNDA into the required plasmid using the preferred cloning strategy (e.i. LIC [23], In-Fusion HD Cloning Technology (Clontech) or traditional restriction).

Note 3. Plasmids where protein expression is under the control of an inducible promoter such as $\mathrm{T} 7 / \mathrm{lacO}$ promoter should be used. It is necessary to use two plasmids with identical or different origins of replication but that provide resistance to different antibiotics. These plasmids should ideally allow the fusion of the HK to two different tags in order to provide short and long versions of the protein. In our study, we used as plasmids pLIC-SGC1 and pNIC28-Bsa4 [23], containing both N-terminal His-tag to produce long versions of the HK, and pET21d and pET24a (Novagene) without tag to produce short versions of the HK.

Note 4. T4 polymerase is required to create overhangs with complementarity between the plasmid and insert in the case of LIC cloning.

Note 5. It is important to choose a suitable E. coli strain for HK expression. It will depend on the presence of rare codons in the HK, if any toxicity is caused to the bacteria during expression and to the level of expression needed. For example, the cell strain BL21-codonplus (DE3)-RIL uses T7 RNA polymerase under the IPTG-inducible lacUV5 promoter to produce high levels of expression. This strain contains a plasmid with chloramphenicol (Clo) resistance that provides extra copies of the tRNA genes that recognize the Arg codons AGA and AGG, the isoleucine codon AUA and the Leu codon CUA. 
Note 6. The $\mathrm{pH}$ of the buffer will depend on the $\mathrm{pl}$ of the protein. Addition of $1 \mathrm{mM}$ of phenylmethylsulfonyl fluoride (PMSF) to inhibit protease activity and $1 \mathrm{mM}$ of $\beta$-mercaptoethanol to avoid oxidation is recommended during lysis.

Note 7. An example of mutated residues involved in ATP binding could be N380A in HK853 and N347A in EnvZ which corresponds to a conserved Asn that comprises the $\mathrm{N}$-box in the $\mathrm{CA}$ domain in charge of binding the $\mathrm{Mg}^{2+}$ ion that stabilizes de ATP. Mutation to Ala of this residue impairs binding to the $\mathrm{Mg}^{2+}$ ion. Note 8. Alternatively, to the conserved Asn in the N-box, other residues could be mutated to Ala in order to avoid ATP binding and impair the kinase activity. However, these other residues should be checked for loss of ATP binding by performing ligand binding assays.

Note 9. The cytoplasmic catalytic portion of some HKs may undergo a phenomenon called spontaneous subunit exchange based on a continuously forming and breaking protein dimer. This phenomenon could affect the stability of the heterodimer formation. This is the case of the HK EnvZ that show trans autophosphorylation where the heterodimer $L^{ø} S$ shows both subunits phosphorylated due to the formation of wild-type $S$ dimer. In this case, it is necessary to produce the heterodimer $\mathrm{L}^{\varnothing} \mathrm{S}^{\varnothing}$ to confirm which subunit has become phosphorylated.

Note 10. To generate the heterodimers, the plasmids used to express the $L$ and $S$ versions of the protein must have resistance to different antibiotics. Thus, the inclusion of both antibiotics in the culture medium will force their maintenance in the $E$. coli strain. It is also advisable that the expression of both proteins is under the control of the same promoter and that both plasmids present a similar number of copies, thus ensuring a similar expression level of $L$ and $S$ versions. 
Note 11. Purification of lower amounts of protein (e.g., produced in $20 \mathrm{ml}$ of culture), can be performed in batch using a $\mathrm{Ni}^{2+}$ High Density resin (ABT) and following the indications of the supplier.

Note 12. Glycerol, sucrose or ethylene glycol, as well as high concentrations (> $30 \%$ ) of polyethylene glycols (PEGs), can be good options as cryoprotectant depending on the crystallization conditions.

Note 13. The role of the catalytic residues proposed in HK853 and EnvZ was determined by their autophosphorylation capacity, but this capacity is directly dependent on the ability to bind ATP. Thus, to be able to distinguish if the contribution of a catalytic residue was solely based on the enzymatic reaction or on the ATP binding it is advisable to check the contribution of the mutation in their ATP binding capacity through Isothermal Calorimetry assays (ITC) or Surface Plasmon resonance (SPR) using AMPPNP, a non-hydrolysable ATP analogue.

\section{Acknowledgements}

This work was supported by Spanish Government (Ministry of Economy and Competitiveness) grants BIO2016-78571-P to A.M and BFU2016-78606-P to P.C. is the recipient of a Ramón y Cajal contract, from the Ministry of Economy and Competitiveness. C.M-M is the recipient of a PhD fellowship from the Progama de becas, Secretaría de Educación Superior, Ciencia, Tecnología e Innovación of Ecuador Government (2015-AR2Q9228)

\section{References}

1. Gao, R. \& A.M. Stock, (2009) Biological insights from structures of twocomponent proteins. Annu Rev Microbiol 63: 133-154. 
2. Dutta, R. \& M. Inouye, (2000) GHKL, an emergent ATPase/kinase superfamily. Trends Biochem Sci 25: 24-28.

3. Marina, A., C. Mott, A. Auyzenberg, W.A. Hendrickson \& C.D. Waldburger, (2001) Structural and mutational analysis of the PhoQ histidine kinase catalytic domain. Insight into the reaction mechanism. J Biol Chem 276: 41182-41190.

4. Bilwes, A.M., C.M. Quezada, L.R. Croal, B.R. Crane \& M.I. Simon, (2001) Nucleotide binding by the histidine kinase CheA. Nat Struct Biol 8: 353-360.

5. Yang, Y. \& M. Inouye, (1991) Intermolecular complementation between two defective mutant signal-transducing receptors of Escherichia coli. Proc Natl Acad Sci U S A 88: 11057-11061.

6. Ninfa, E.G., M.R. Atkinson, E.S. Kamberov \& A.J. Ninfa, (1993) Mechanism of autophosphorylation of Escherichia coli nitrogen regulator II (NRII or NtrB): transphosphorylation between subunits. J Bacteriol 175: 7024-7032.

7. Casino, P., V. Rubio \& A. Marina, (2009) Structural insight into partner specificity and phosphoryl transfer in two-component signal transduction. Cell 139: 325-336.

8. Casino, P., V. Rubio \& A. Marina, (2010) The mechanism of signal transduction by two-component systems. Current opinion in structural biology 20: 763-771.

9. Ashenberg, O., A.E. Keating \& M.T. Laub, (2013) Helix bundle loops determine whether histidine kinases autophosphorylate in cis or in trans. J Mol Biol 425: 1198-1209.

10. Casino, P., L. Miguel-Romero \& A. Marina, (2014) Visualizing autophosphorylation in histidine kinases. Nat Commun 5: 3258. 
11. Cai, S.J. \& M. Inouye, (2003) Spontaneous subunit exchange and biochemical evidence for trans-autophosphorylation in a dimer of Escherichia coli histidine kinase (EnvZ). J Mol Biol 329: 495-503.

12. Mechaly, A.E., N. Sassoon, J.M. Betton \& P.M. Alzari, (2014) Segmental helical motions and dynamical asymmetry modulate histidine kinase autophosphorylation. PLOS Biol 12: e1001776.

13. Letunic, I., T. Doerks, and P. Bork. (2015) SMART: Recent Updates, New Developments and Status in 2015. Nucleic Acids Research 43(D1): D257-60. 14. Finn, R.D., Coggill, P., Eberhardt, R.Y., Eddy, S.R., Mistry, J., Mitchell, A.L., Potter, S.C., Punta, M., Qureshi, M., Sangrador-Vegas, A., Salazar, G.A., Tate, J., Bateman, A. (2016) The Pfam protein families Database: towards a more sustainable future. Nucleic Acids Res. Jan 4;44(D1):D279-85.

15. Savitsky, P., J. Bray, C.D. Cooper, B.D. Marsden, P. Mahajan, N.A. BurgessBrown \& O. Gileadi, (2010) High-throughput production of human proteins for crystallization: the SGC experience. J Struct Biol 172: 3-13.

16. McPherson A \& Gavira J. A. (2014) Acta Crystallogr F Struct Biol Commun Jan;70(Pt 1):2-20.

17. Winn, M.D., C.C. Ballard, K.D. Cowtan, E.J. Dodson, P. Emsley, P.R. Evans, R.M. Keegan, E.B. Krissinel, A.G. Leslie, A. McCoy, S.J. McNicholas, G.N. Murshudov, N.S. Pannu, E.A. Potterton, H.R. Powell, R.J. Read, A. Vagin \& K.S. Wilson, (2011) Overview of the CCP4 suite and current developments. Acta Crystallogr D Biol Crystallogr 67: 235-242.

18. Adams, P.D., P.V. Afonine, G. Bunkoczi, V.B. Chen, I.W. Davis, N. Echols, J.J. Headd, L.W. Hung, G.J. Kapral, R.W. Grosse-Kunstleve, A.J. McCoy, N.W. Moriarty, R. Oeffner, R.J. Read, D.C. Richardson, J.S. Richardson, T.C. 
Terwilliger \& P.H. Zwart, (2010) PHENIX: a comprehensive Python-based system for macromolecular structure solution. Acta Crystallogr D Biol Crystallogr 66: 213221.

19. Panjikar S., V. Parthasarathy, V.S. Lamzin, M.S. Weiss, P.A Tucker. (2005) Auto-rickshaw: an automated crystal structure determination platform as an efficient tool for the validation of an X-ray diffraction experiment. Acta Crystallogr D Biol Crystallogr. 61(Pt 4):449-57.

20. Adams PD, Grosse-Kunstleve RW, Hung LWet al (2002) PHENIX: building new softwarefor automated crystallographic structure deter-mination. Acta Crystallogr D Biol Crystallogr58:1948-195421.

21. Collaborative Computational Project, Number4 (1994) The CCP4 suite: programs for pro-tein crystallography. Acta Crystallogr D BiolCrystallogr 50:76076322.

22. Emsley P, Cowtan K (2004) Coot: model-building tools for molecular graphics. ActaCrystallogr D Biol Crystallogr 60:2126-2132

23. WL DeLano (2002) The PyMOL moleculargraphics system, version 11, Schro dinger LLChttp://wwwpymolorg24. Murshudov GN, Vagin 


\section{Figure captions}
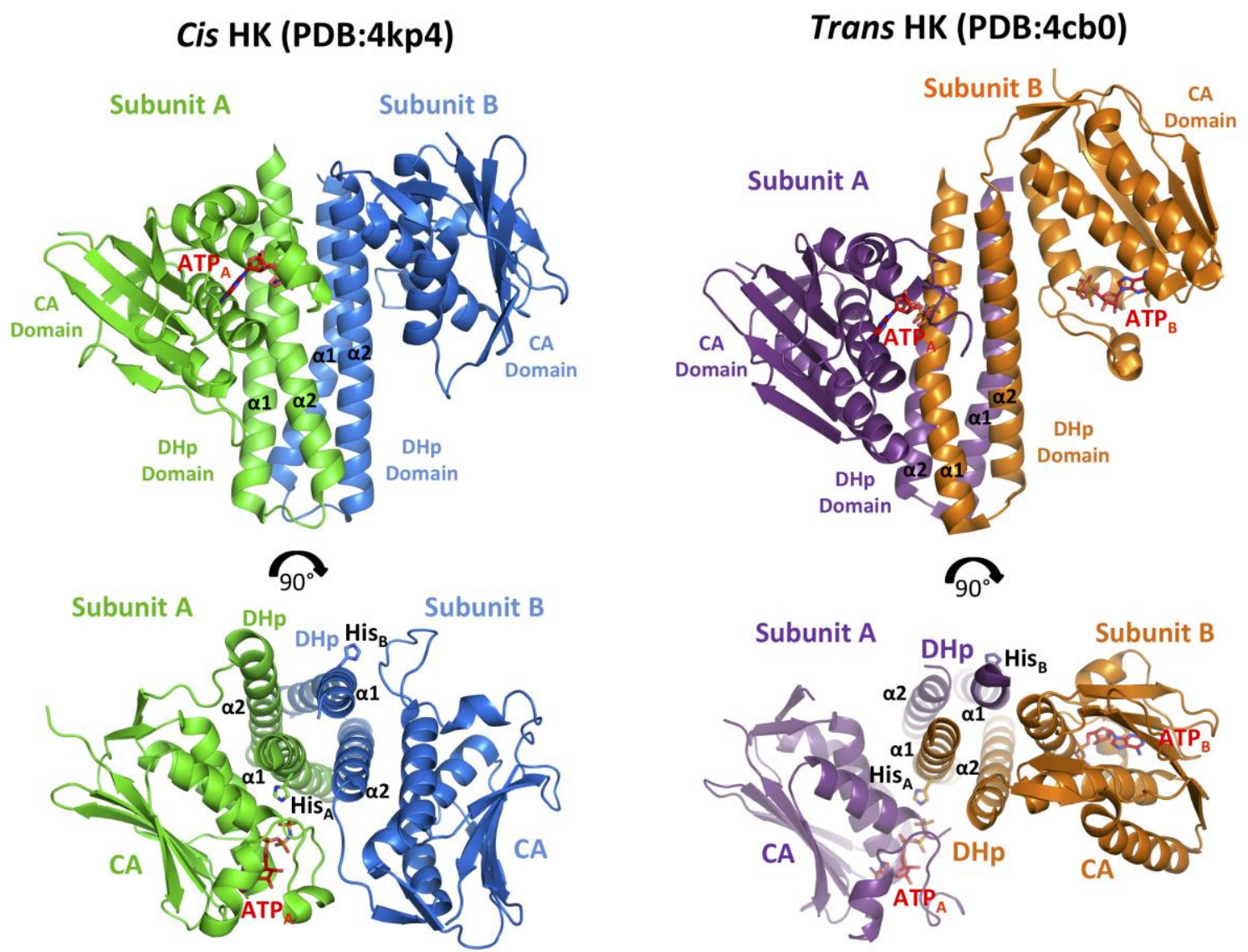

Figure 1. Cis and trans autophosphorylation. 3D structures of the catalytic domains of HKs showing the conformation for autophosphorylatio. Two orthogonal views of EnvZ ${ }^{\text {chim }}$ (PDB:4k4p) showing cis autophosphorylation and CpxA (PDB:4cb0) showing trans autophosphorylation are represented in cartoon. In the dimers, each subunit is represented in a different color, green and blue for the cis HK subunits and orange and purple for the trans HK subunits. The ATP ligands are represented in stick with carbons in red. The DHp and CA domains conforming the HK catalytic domains are labelled as well the $\alpha 1-\alpha 2$ helices and phosphoaceptor His in the DHp domains. 


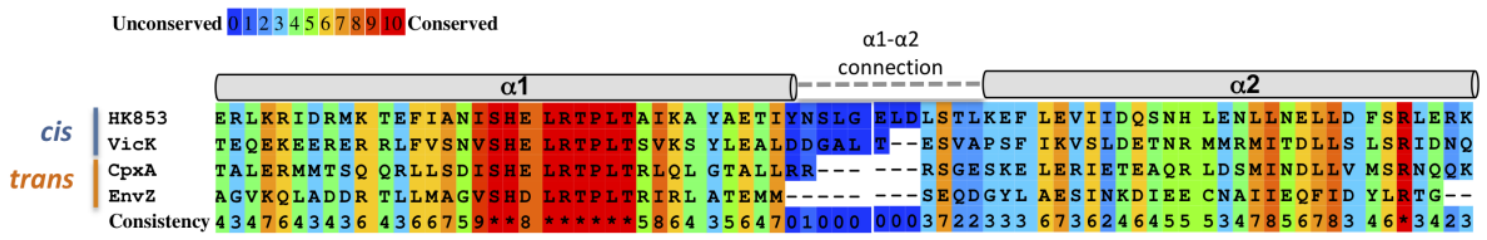

Figure 2. Sequence alignment of the DHp. The sequences of DHp domains from HKs with cis, HK853 and Vick, and trans, EnvZ and CpxA, autophosphorylation are aligned. The alignment was done with PRALINE webserver and the code of colours for residue conservation is in the top of the figure. The secondary structure elements of the DHp is shown at the top of the alignment. The sequence and size variable connector between $\alpha 1-\alpha 2$ helices is indicated. 
A

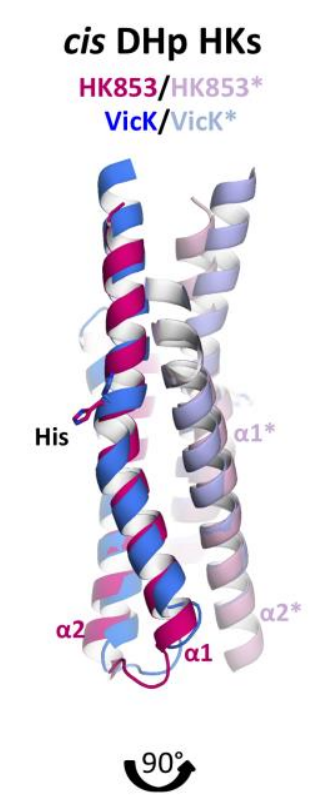

Counter-clockwise

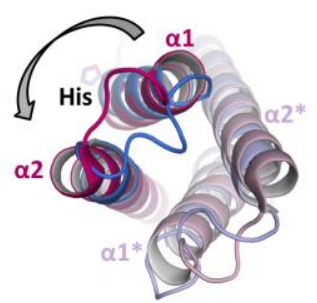

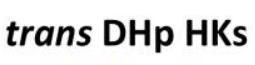

EnvZ/EnvZ*

СрхА/СpхA*

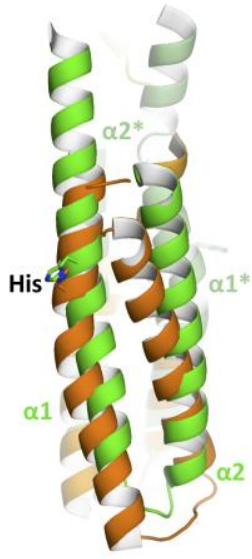

90.

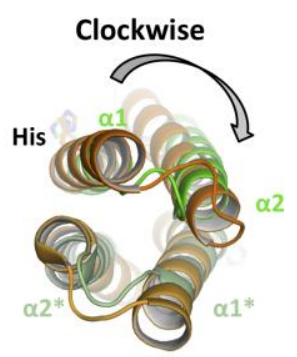

B

cis-trans DHp HKs

HK853/HK853*

EnvZ/EnvZ*
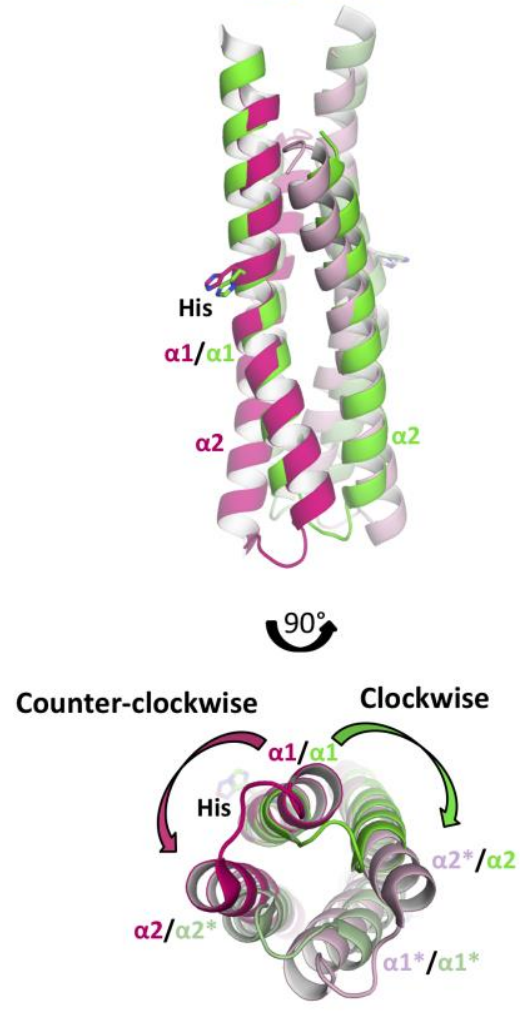

Figure 3. Directionality of the $\alpha 1-\alpha 2$ DHp connection in HKs. A) Superimposition of DHp domains from HK853 (red tones; PDB: 3DGE) and VicK (blue tones; PDB: 4I5S) HKs which show cis autophosphorylation and from EnvZ (green tones; PDB: 4CTI) and CpxA (orange tones; PDB: 4BIU) that show trans autophosphorylation. B) Superimposition of $\mathrm{DHp}$ domains of cis and trans autophosphorylation HKs, HK853 and EnvZ respectively. At the bottom of the panel is represented an apical view of the superimposed structures showing counter-clockwise or clockwise direction of the $\alpha 1-\alpha 2$ DHp connection. 
A

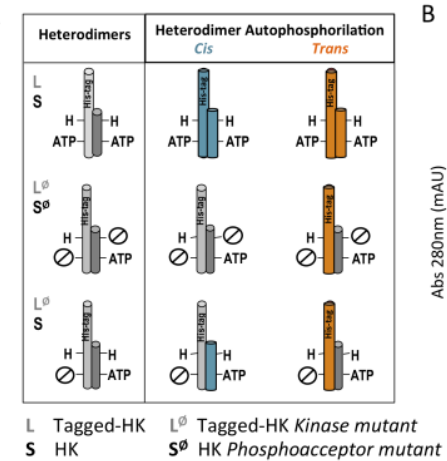

S HK $S^{\oplus}$ HK Phosphoacceptor mutant

C

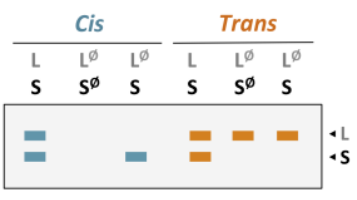

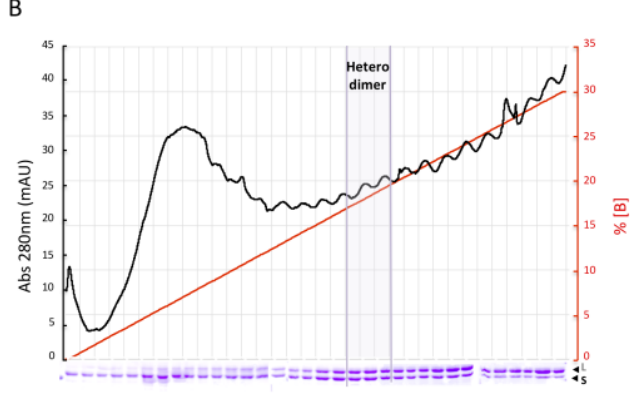

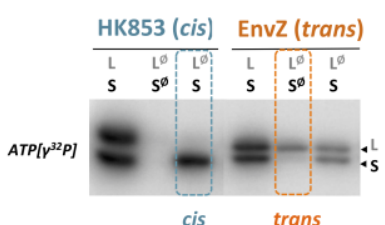

Figure 4. Heterodimer formation. A) Schematic representation of heterodimer species labeling the subunits accordingly to the absence ( $L$ and $S$ ) or presence $\left(\mathrm{L}^{\Phi}\right.$ and $\left.\mathrm{S}^{\Phi}\right)$ of mutation is shown in the left side of the scheme. In the right side the phosphorylated subunits are coloured in each heterodimer as if the autophosphorylation reaction was carried out in cis (blue colour) or in trans (orange colour). B) Example of affinity chromatography for heterodimer purification. The chromatogram corresponds to a 5-ml HisTrap FF column eluted with a gradient of buffer B (red line) in 20 column volumes Samples from each $3 \mathrm{ml-fraction}$ were loaded in a 15\% SDS-PAGE gel and those fractions containing an equal ratio of $L$ and $S$ subunits as heterodimer (region in purple shadow) were selected. C) Visualization of heterodimer autophosphorylation. Left, Schematic representation of autophosphorylation patters with samples obtained in A) in a hypothetical SDS-PAGE gel. Right, autorradioagraphy of an experiment carried out with the heterodimers for HK853 (cis) and EnvZ (trans). Phosphorylation of $L^{\Phi}$ subunit in $\mathrm{L}^{\Phi} S^{\Phi}$ heterodimer allows to identify the trans autophosphorylation (in blue) while phosphorylation in $S$ subunit in $L^{\Phi} S$ heterodimer allow to identify the cis autophosphorylation (in orange). 
A

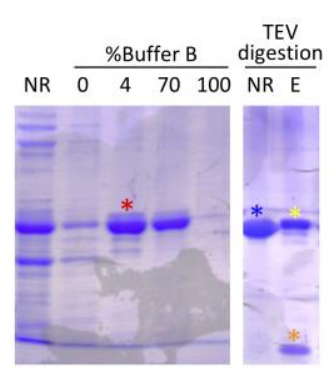

C

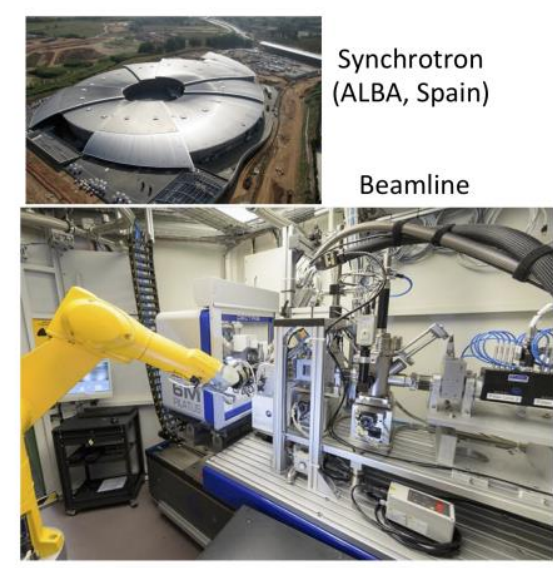

96-Wells MRC2 plate
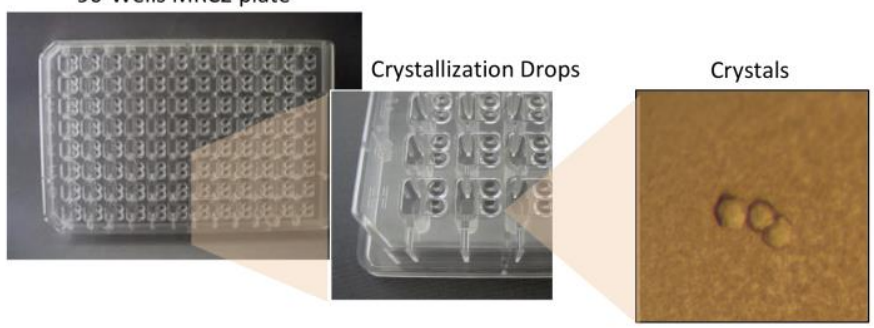

Diffraction pattern

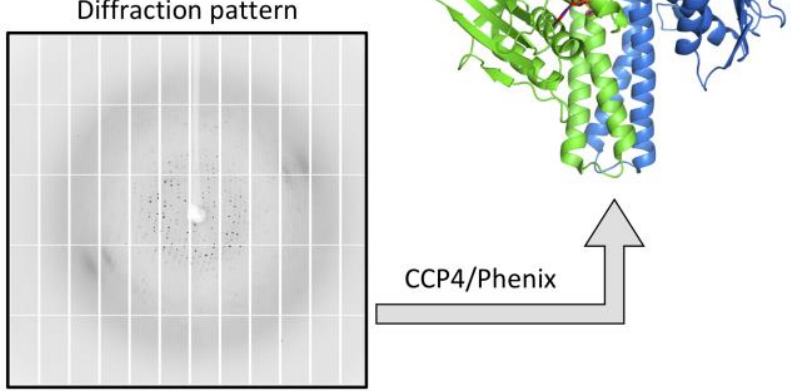

Figure 5. Scheme of the steps to determine the 3D structure of a HK by Xray crystallography. A) SDS PAGE showing the purification steps of the HK before and after removal of the tag. Red and blue asterisks indicate the bang corresponding to the HK with and without tag, respectively. Yellow and orange asterisks indicate the bands corresponding to the TEV polymerase and the tag, respectively, that elute with $100 \%$ of buffer B. B) MRC2 plates for protein crystal growth in an appropriate screening. C) Pictures of synchrotron (ALBA, Spain) and X-ray beamline (XALOC in ALBA) used for protein crystals diffraction. Images containing diffraction patterns are processed with computer suites such as CCP4 of Phenix to obtain the final 3D structure of the HK. 
A

HK853

EnvZ

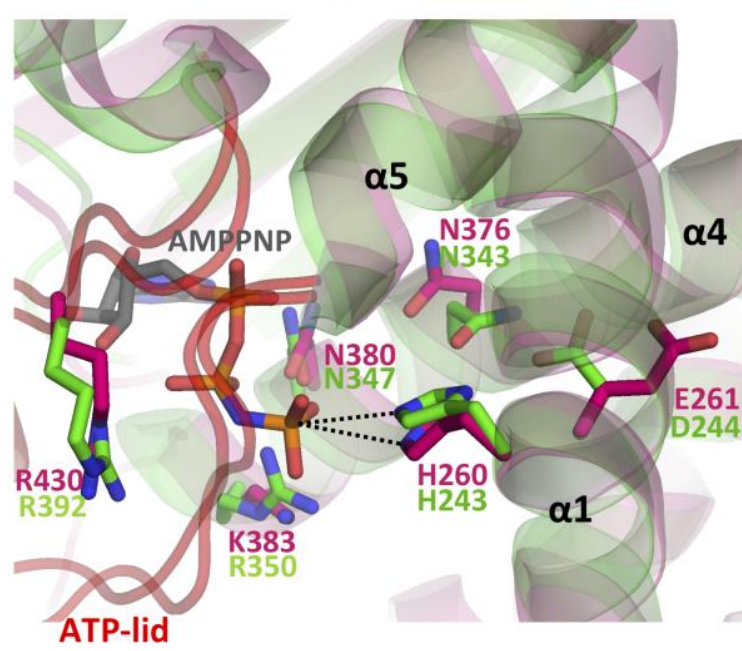

B

$A T P\left[\gamma^{32} P\right]$

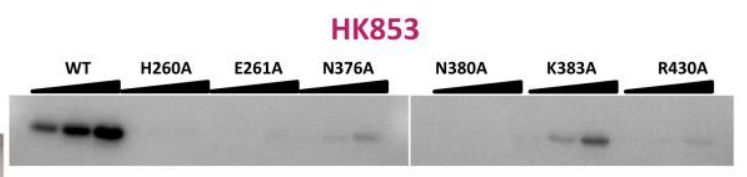

EnvZ

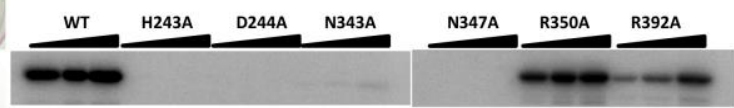

Figure 6. Residues involved in the catalytic mechanism of the autophosphorylation reaction. A) Detail of the active center in the 3D structure of EnvZ $Z^{\text {chim }}$ (in green) showing the close distance between AMPPNP (in sticks with carbom and phosphate atoms in grey and orange, respectively) and the phosphoacceptor His. Superimposed DHp and CA domains of HK853 over EnvZ $Z^{\text {chim }}$ structure are shown in pink. Catalytic relevant residues are drawn as sticks and labelled. B) Autophosphorylation activity assays of Ala mutants in relevant catalytic residues of $\mathrm{HK} 853$ and EnvZ. 